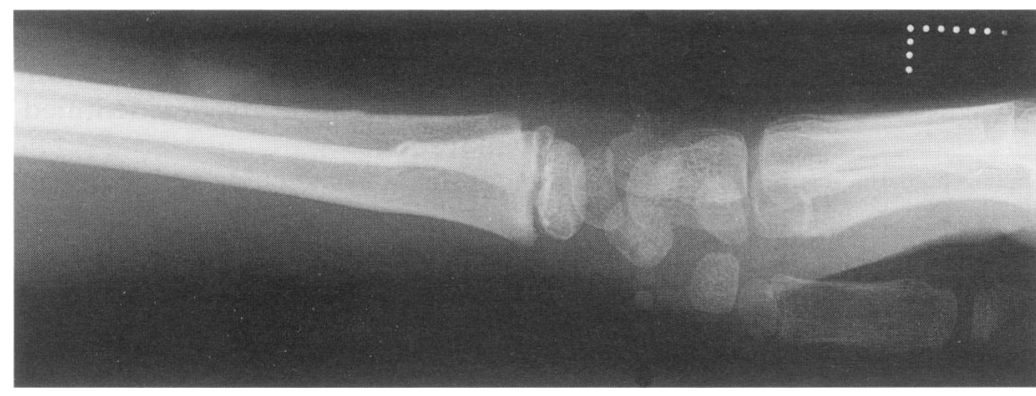

Fig $1 X$ rays of the left wrist demonstrating the initial greenstick fracture.

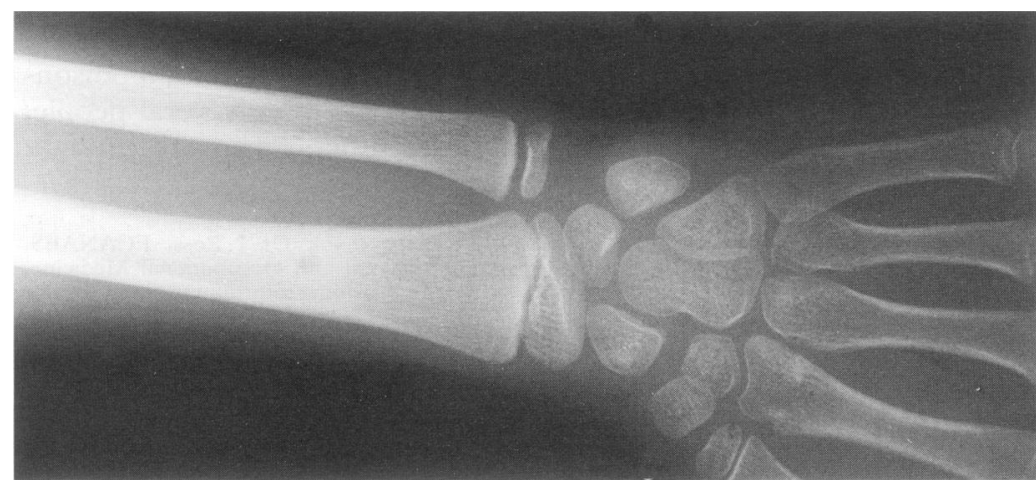

Fig $2 X$ rays after reinjury showing a well defined cystic lesion adjacent to the healed greenstick fracture. slightly oval shaped, and may be multiple. ${ }^{2}$ Healing of the initial fracture is not affected by these cysts, nor do they predispose to pathological fractures. Pfister-Goedeke and Braune reported spontaneous resolution in three out of nine cases $(33 \%)$ at 3 years. ${ }^{3}$

There is debate about the aetiology of these cysts. Pfister-Goedeke and Braune ${ }^{3}$ suggested that they are resorption cysts within an excessive periosteal reaction, related to the subperiosteal haematoma that accompanies greenstick fractures. Phillips and Keats attribute post-traumatic cysts to the resorption of intraosseous haemorrhage. ${ }^{4}$ Malghem et al reported that computed tomographic scanning of two cases showed a density consitent with a fatty content. ${ }^{5}$ They postulated that this fat resulted from the inclusion of medullary fat within the subperiosteal haematoma. This theory of transcortical escape of intramedullary fat is supported by Davids et al, who showed fat within a postfracture cortical cyst on magnetic resonance imaging. ${ }^{1}$ Uncertainty about the aetiology of these lesions is likely to continue as long as their histological nature remains unknown. ${ }^{6}$ However, to date, biopsy of these lesions has never been clinically justified in view of their asymptomatic nature.

These small asymptomatic postfracture cortical cysts are invariably incidental $x$ ray findings and of little clinical importance. Failure to recognise this apparently rare complication of minor fractures in children may cause confusion for the clinician and un necessary concern for parents. Expensive diag nostic investigation or operative intervention are not indicated in these cysts. We advocate review after 6 to 12 months to ensure that the lesion remains asymptomatic. ally been reported. A review of the English language reports ${ }^{1}$ identified 17 similar cases, 15 involving the distal radial metaphysis. The incidence of these postfracture cysts is unknown. They are probably more common than the published reports would suggest since most distal radius fractures in children are monitored on clinical signs alone.

In all previously reported cases the postfracture cortical cysts were asymptomatic. These cysts are discovered either during radiological follow up of a fracture or after reinjury of the same limb (as in this case). They usually appear more than one month after the fracture. Characteristically the cysts are less than $10 \mathrm{~mm}$ in diameter, do not expand, appear rounded or
1 Davids JR, Graner KA, Mubarak SJ. Post-fracture lipid inclusion cyst. $\mathcal{F}$ Bone foint Surg (Am) 1993;75:1528-32.

2 Malghem J, Maldague B. Transient fatty cortical defects following fractures in children. Skel Radiol 1986;15: 368-71.

3 Pfister-Goedeke L, Braune M. Cyst-like cortical defects following fractures in children. Paediatr Radiol 1981;11: 83-6.

4 Phillips CD, Keats TE. The development of post-traumati cyst-like lesions in bone. Skel Radiol 1986;15:631-4.

5 Malghem J, Maldague B, Claus D, Clapuyt P. Transient cyst-like cortical defects following fractures in children. f Bone foint Surg (Br) 1990;72:862-5.

6 Moore TE, King AR, Travis RC, Allen BC. Post-traumatic cysts and cyst-like lesions of bone. Skel Radiol 1989;18: 93-7.

\title{
Two cases of paraduodenal hernia, a rare internal hernia
}

\author{
Department of \\ Emergency Medicine, \\ Fremantle Hospital, \\ PO Box 480 \\ Western Australia 6160 , \\ Australia \\ T McDonagh \\ G A Jelinek \\ Correspondence to: \\ G A Jelinek MD.
}

Thomas McDonagh, George A Jelinek

\author{
Abstract \\ Paraduodenal hernia is a rare congenital \\ internal hernia which arises from an error \\ of rotation of the midgut with entrapment \\ of the small intestine beneath the develop-
}

ing colon. It is important as it usually presents as intestinal obstruction, and before laparotomy is often misdiagnosed. Mortality increases significantly with delays in surgical treatment. Two cases 
are presented: an 8 year old boy and a 52 year old man. Both presented with a short history of abdominal pain and an acute abdomen. With prompt surgical treatment, they recovered rapidly.

( $\mathcal{A}$ Accid Emerg Med 1996;13:64-68)

Key terms: abdominal pain; acute abdomen; interna hernia; intestinal obstruction; paraduodenal hernia.

Paraduodenal hernia, also referred to as internal, congenital, retroperitoneal, or mesocolonic hernia, is a herniation resulting from anomalies of rotation and reduction of the midgut loop in the embryo. This hernia occurs when the small intestine becomes trapped beneath the colon because of anomalous rotation of the mesentery of the developing colon. The duodenum and small intestine become trapped in a sac lined by peritoneum, behind the mesentery of the colon, either to the left or to the right of the midline. ${ }^{1-3}$

We present two patients who required laparotomy because of intestinal obstruction from paraduodenal hernia.

\section{Case 1}

An eight year old boy presented to Fremantle Hospital with a $5 \mathrm{~h}$ history of abdominal pain which developed suddenly in the left upper abdomen, associated with vomiting. Previously he had had three similar episodes, though never as severe. These were sudden in onset, lasted a few hours, and settled spontaneously with rapid relief of pain. Each had been associated with vomiting. Two weeks before presentation he had suffered a viral illness.

On arrival he was ashen grey in colour, cool and peripherally constricted. Blood pressure was $70 \mathrm{~mm} \mathrm{Hg}$ systolic, pulse 140 beats $/ \mathrm{min}$, and temperature $34 \cdot 2^{\circ} \mathrm{C}$. Conscious state was depressed but he was able to respond to commands. Abdominal examination revealed

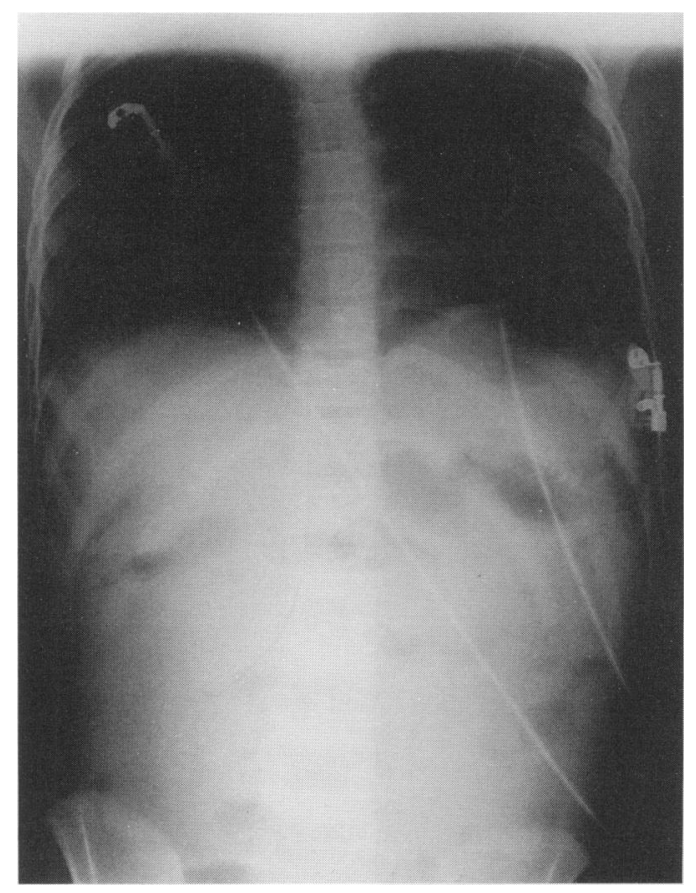

Fig 1 Abdominal x ray, case 1. marked tenderness with guarding in the left side of the abdomen. Bowel sounds were increased. After a fluid bolus of $1000 \mathrm{ml}$ of normal saline, blood pressure was $100 \mathrm{~mm} \mathrm{Hg}$ systolic and pulse $120 / \mathrm{min}$.

A portable abdominal $x$ ray (fig 1 ) revealed an isolated gas filled loop of bowel. White cell count was $31.3 \times 10^{9} /$ litre with $25 \times 10^{9} /$ litre neutrophils. Electrolytes, amylase, and liver function tests were normal.

A provisional diagnosis of hypovolaemic shock secondary to volvulus, intussusception, or splenic rupture following viral illness was made. Laparotomy revealed a paraduodenal hernia with herniation of $30 \mathrm{~cm}$ of mid-jejunum into a left paraduodenal hernial sac. The incarcerated loop was ischaemic and was reduced with difficulty, returning normal perfusion to the bowel. The mesenteric defect was then closed. Postoperative course was uneventful and he was discharged home on day seven.

\section{Case 2}

A 52 year old man was transferred to Fremantle hospital emergency department from a peripheral hospital, having presented after $5 \mathrm{~h}$ of abdominal pain. The pain was sudden in onset, severe, and initially across the upper abdomen. It later became generalised and constant. The patient vomited four times but passed no flatus.

He had been recently well, with a history of controlled angina, raised cholesterol, and a cholecystectomy 12 years earlier. Medications were aspirin, diltiazem, and cholestyramine.

On examination the patient appeared unwell, dehydrated, and distressed. $\mathrm{He}$ was afebrile, with a blood pressure of $130 / 70 \mathrm{~mm}$ $\mathrm{Hg}$ and pulse 80 beats $/ \mathrm{min}$. Abdominal examination revealed generalised guarding and rebound tenderness. Bowel sounds were absent.

White cell count was $21.9 \times 10^{9} /$ litre with neutrophils $18.5 \times 10^{9} /$ litre. Electrolytes were normal, and amylase was not raised. Blood sugar was $15 \cdot 1 \mathrm{mmol} /$ litre. Abdominal $x$ rays (figs 2 and 3) revealed slightly distended loops of upper small bowel.

Following intravenous fluid replacement and narcotic analgesia, laparotomy showed small bowel obstruction because of a paraduodenal hernia at the duodenal-jejunal flexure. The sac was anterolateral to the duodenal-jejunal flexure and extended to the left to the descending colon and contained the left colic artery in its edge. The small bowel was viable and was reduced from the hernial sac. The sac was obliterated by suturing the posterior peritoneum.

The patient was discharged four days after admission. At review in clinic he was well. Subsequent investigation confirmed the presence of diabetes which has been controlled with a diabetic diet.

\section{Discussion}

The cases illustrate the typical onset of bowel obstruction caused by paraduodenal hernia, with rapidly progressing acute abdominal pain from ischaemic bowel. As it is a closed loop 


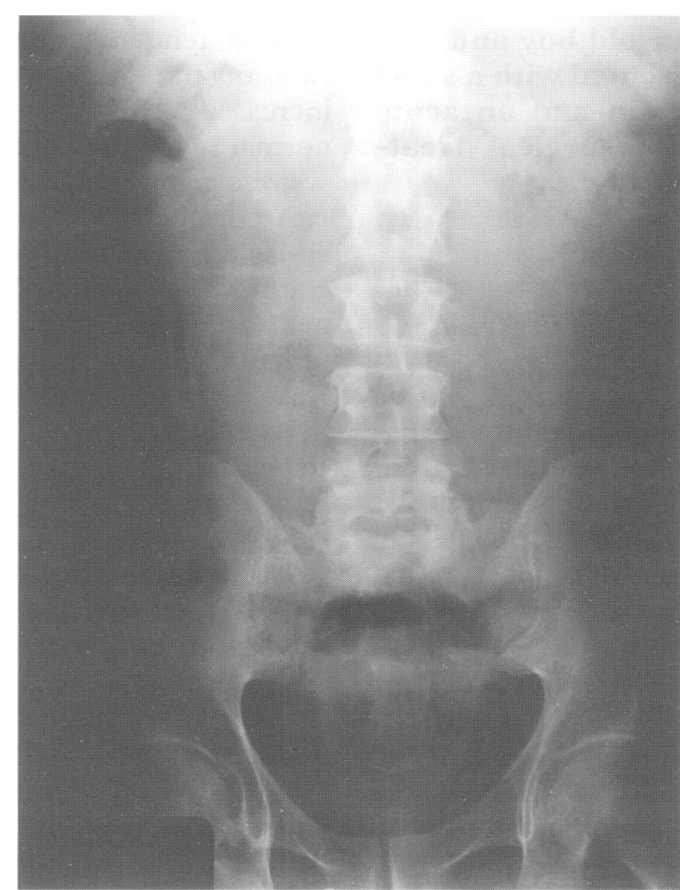

Fig 2 Supine abdominal x ray, case 2.

obstruction, damage to the bowel occurs early, accounting for the high mortality of this condition. Presentation is dramatic, often with hypovolaemic shock and peritonism.

In the first case, the history was typical with multiple previous episodes and dramatic onset. The diagnosis was not made preoperatively because of non-specific symptoms, signs, and abdominal $x$ ray findings.

\section{PATHOPHYSIOLOGY}

Paraduodenal hernia can be divided into two types, left and right (fig 4). A left sided hernia (fig 5) is produced by malrotation of the umbilical loop. As the small intestine is reduced

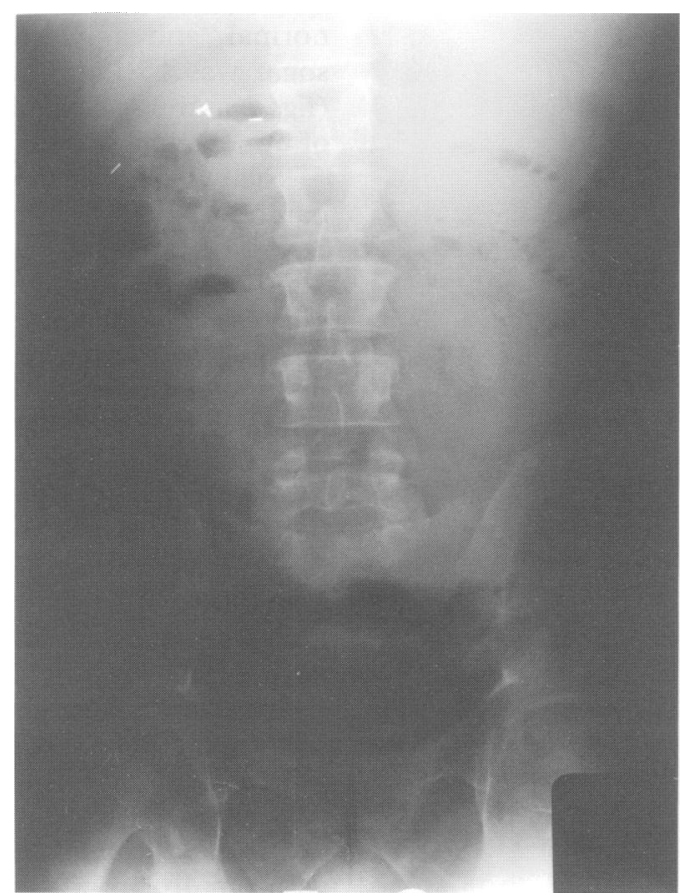

Fig 3 Erect abdominal $x$ ray, case 2.

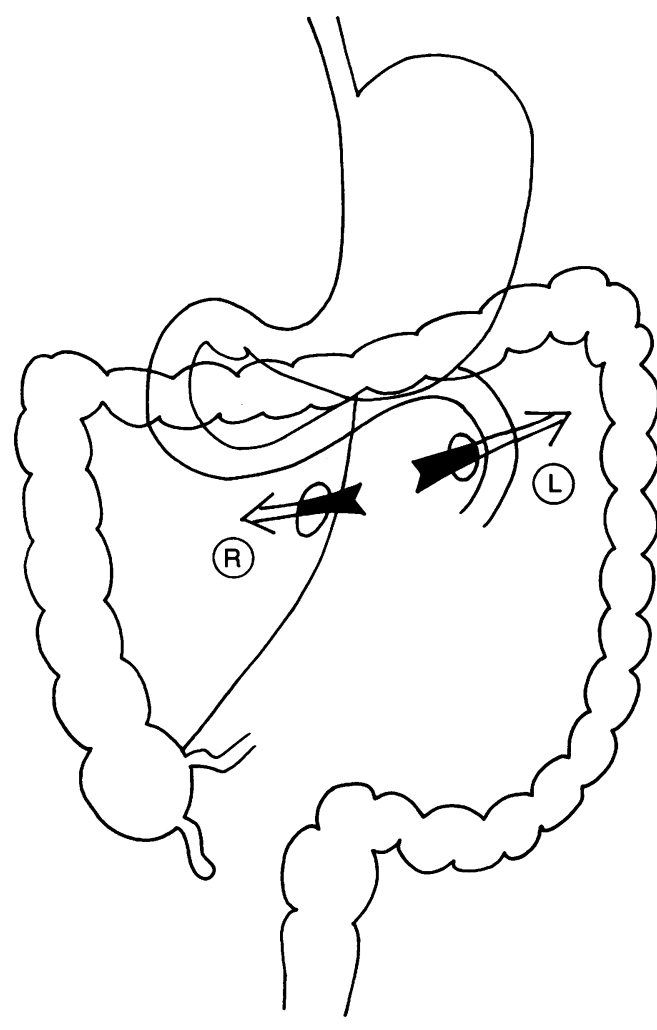

Fig 4 Left and right paraduodenal hernia.

into the abdominal cavity, it is caught beneath the mesentery of the descending colon. The caecum is completely rotated and lies in the normal position in the right iliac fossa. The sac points downward and to the left towards the descending colon. The free edge of the sac

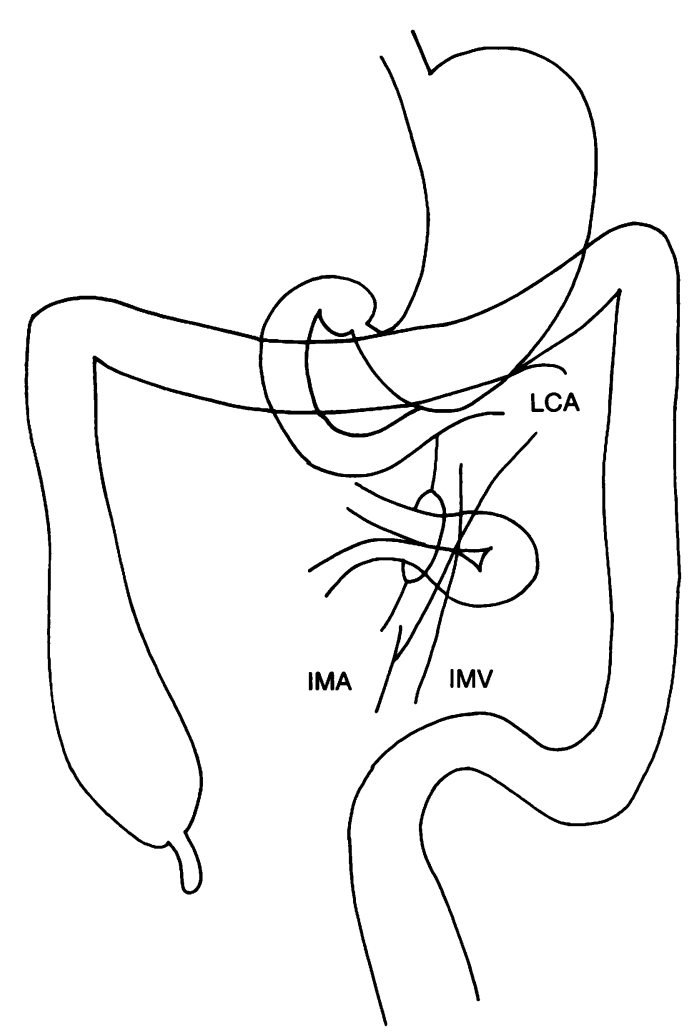

Fig 5 Left paraduodenal hernia.

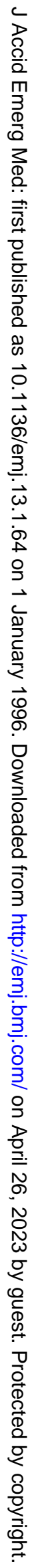


contains the inferior mesenteric artery and vein and the left colic artery, and their branches are an integral part of the hernia sac. The anterior wall of the sac is made up of the mesocolon of the descending colon. ${ }^{1-6}$

A right paraduodenal hernia (fig 6) is produced when the umbilical loop fails to rotate completely and is caught beneath the caecum. The small intestine becomes imprisoned behind its mesentery. The major portion of the small intestine remains to the right of the superior mesenteric artery. The anterior wall of the sac is made up of the mesentery of the ascending colon and transverse colon. Right sided paraduodenal hernias contain the superior mesenteric artery and iliocolic artery in the free edge of the sac. The hernial orifice is to the right of midline and the peritoneal sac is directed downward and to the right. ${ }^{1-6}$

\section{EPIDEMIOLOGY}

Internal hernias account for $0.5 \%$ of all cases of intestinal obstruction. ${ }^{7}$ Paraduodenal hernia is the most common type, accounting for $53 \%$ of cases. ${ }^{1-11}$ Paraduodenal hernia can present at any age but typically is seen between the fourth and sixth decades of life. ${ }^{11}{ }^{12}$ It is more common in men than women in a ratio of $3: 1,11012$ and more frequent on the left than right in a ratio of $3: 1 .^{2}$ Fifty per cent of paraduodenal hernias cause obstruction; the remainder are diagnosed incidentally at laparotomy or at necropsy. ${ }^{1011}$

\section{CLINICAL FEATURES}

Small and easily reducible hernias may remain asymptomatic during life. Clinical manifestations result from partial or complete obstruction of the small intestine. ${ }^{189} 11$

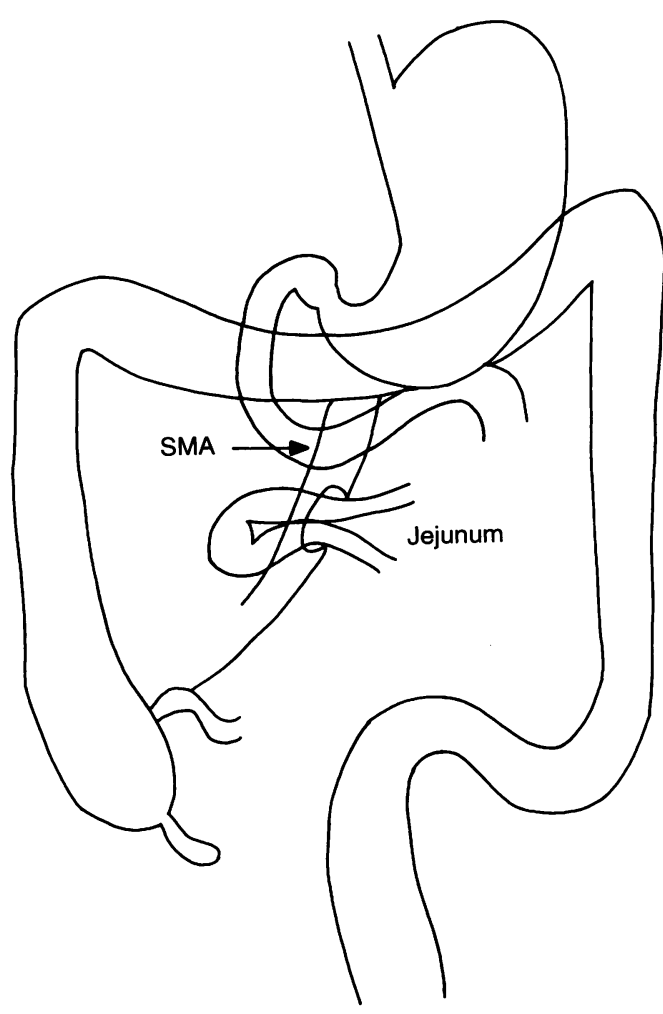

Fig 6 Right paraduodenal hernia.
Most patients experience intermittent colicky abdominal pain in the periumbilical or epigastric regions or complain of chronic indigestion. Episodes of nausea, vomiting, and obstructive symptoms may occur, especially after a large meal. The pain may be posturally related, being worse on standing or exertion and relieved by lying down. The recurrent attacks of abdominal pain may resemble those of peptic ulceration, biliary disorders, or abdominal angina. ${ }^{13}$ Seventy to eighty per cent of patients have a long history of symptoms lasting decades, and the pain may be labelled as functional before the condition is finally diagnosed. ${ }^{7}$

Only rarely is the diagnosis made preoperatively. Physical examination during a symptom-free period is likely to be normal. When the hernia is incarcerated, physical examination is usually not diagnostic. Occasionally a palpable mass representing the small bowel loops contained within the hernial sac is present in association with local or diffuse abdominal tenderness. ${ }^{7}{ }^{14}$

The possibility of an internal hernia should be considered in cases of acute or intermittent small bowel obstruction, particularly in the absence of external hernias or history of previous abdominal surgery. The diagnosis may not be evident at laparotomy because of a spontaneous reduction of the hernia or inadvertent reduction at surgery by traction on the small bowel, or because the usual exploratory laparotomy is inadequate for evaluation of the peritoneal fossae.

Gastrointestinal contrast studies reveal the most diagnostic features, including abnormal location of the small intestine, sacculation and crowding of loops of small intestine contained in the hernial sac which does not alter with position, and segmental dilatation and prolonged stasis in the herniated bowel. ${ }^{3914}$ Computerised tomography may show a sac-like mass of dilated loops of small intestine which is retroperitoneal in location. ${ }^{12} 13$

The majority of patients with paraduodenal hernia present with a closed loop obstruction, where the time interval between obstruction and necrosis of bowel may be very short. Any time consuming diagnostic work-up before surgery may endanger life. ${ }^{11}$

\section{TREATMENT}

The mortality of obstructed internal herniae exceeds $50 \%,{ }^{8}{ }^{10}$ reflecting the large proportion of patients who present with intestinal obstruction and ischaemia requiring emergency surgery. ${ }^{181415}$ Consequently, all paraduodenal hernias, even those diagnosed incidentally, should be repaired. ${ }^{1} 2814$

\section{CONCLUSION}

Paraduodenal hernia presents dramatically, causes a non-specific clinical picture, and often reduces spontaneously, leading to diagnostic difficulties. Although a rare cause of intestinal obstruction, it has a high mortality. The diagnosis should particularly be borne in mind in the case of intestinal obstruction in patients who have not had previous abdominal surgery or external herniae. 
1 Bernard RS. Paraduodenal hernias. Surg Gynaecol Obstet 1981;152:99-110

WH. The surgical management of paraduodenal hernia. Ann Surg 1968;108: ment of

3 Meyers MA. Paraduodenal hernias: radiologic and arteriographic diagnosis. Radiology 1970;95:29-37.

4 Tirel M. Left paraduodenal hernia. Br $\mathcal{F}$ Surg 1982;69. 114.

5 Filtzer $H$, Sedwick CE. Strangulated paraduodenal hernia: a case report. Surg Clin North Am 1973;53: $371-4$

6 Wilwerth BM, Zollinger RM, Izand RJ. Congenital mesocolic (paraduodenal) hernia: embryologic basis of repair. Am f Surg 1974;128:358-61.

7 Lee TKY, Voon FCT, Chow KW, et al. Unusual variant of right paraduodenal hernia. Aust NZ f Surg 1990;60: 483-5.

8 Freud H, Berlatzky Y. Small paraduodenal hernias. Arch Surg 1977;112:1180-3.
9 Ghahreman GG. Internal abdominal hernias. Surg Clin North Am 1984;64:393-401.

10 Newsom BD, Kukora JS. (1986) Congenital and acquired hernias: unusual causes of small bowel obstruction. $A m$ f Surg 1986;152:279-85.

11 Brigham RA, Saunders JR, Harmon JW, et al. Paraduodenal hernia: diagnosis and management. Surgery 1984;96: 498-502.

12 Olazabal A, Guasch I, Casas D. Case report: CT diagnosis of non-obstructive left paraduodenal hernia. Clin Radio 1992;46:288-9.

13 Turley K. Right paraduodenal hernia. A source of chronic abdominal pain in the adult. Arch Surg 1979;114: 1072-4.

14 Dengler WC, Reddy PP. Right paraduodenal hernia in childhood. A case report. $f$ Pediatr Surg 1989;24: 1153-4.

15 Suflan S, Matsumoto T. Intestinal obstruction. Am ₹ Surg 1975;130:9-14.

\title{
Respiratory compromise: a rare complication of transcutaneous electrical nerve stimulation for angina pectoris
}

\author{
C J Mann
}

\begin{abstract}
Electrical stimulation of any muscle group may produce tetany. If the intercostal muscles are involved this may lead to respiratory embarrasment. A case is presented in which transcutaneous electrical nerve stimulation (TENS) treatment for angina pectoris resulted in respiratory arrest.
\end{abstract}

( $\mathcal{F}$ Accid Emerg Med 1996;13:68-69)

Key terms: transcutaneous electrical nerve stimulation; respiratory arrest.

Electrical neurostimulation as an analgesic technique has been used succesfully for many years particularly in obstetrics. More recently it has been used in patients with severe unstable angina refractory to pharmocological therapy. ${ }^{1-3}$ One potential hazard is highlighted by this case.

\section{Case report}

A 70 year old man with a long history of ischaemic heart disease suffered an attack of angina pectoris during ambulance transport to a routine cardiology outpatient appointment. One year earlier a transcutaneous nerve stimulator system (TENS) had been fitted, with stimulating electrodes placed anteroposteriorly across the chest wall to control the pain of his unstable angina The episode of chest pain in the ambulance had been very severe and he had increased the TENS current to maximum $(50 \mathrm{~mA})$. The ambulance crew brought him directly to the accident and emergency department.

On examination in the resuscitation room the patient was conscious but unable to speak. The respiratory rate was $25 / \mathrm{min}$ with very shallow breaths. There was central cyanosis. On auscultation breath sounds were normal although air entry was minimal. The pulse was $65 / \mathrm{min}$ of good volume and regular. Blood pressure was $140 / 85 \mathrm{~mm} \mathrm{Hg}$.

High flow oxygen was given from a reservoir system with no clinical benefit. It was not possible to record an electrocardiograph as discharges from the TENS machine produced considerable electrical interference. The patient deteriorated and chest wall movement virtually ceased. The respiratory pattern was that of tetanic paralysis with incoordinated sustained muscular contraction.

The TENS electrodes were removed. Immediately the patient exhaled and was able to respire with much greater movement of the chest wall. Within a few minutes the respiratory pattern had returned to normal. On questioning he was pain-free. The dyspnoea subsequently resolved completely. An ECG showed no changes from previous electrocardiographs and after attending the outpatient clinic the patient was discharged home.

\section{Discussion}

The lack of familiarity with neurostimulators for the treatment of angina pectoris meant that the medical staff treating this patient were at a disadvantage in determining the cause of the acute deterioration. There are no previous reports of similar problems with TENS systems, although subjective symptoms of difficulty in breathing are not uncommon (Mannheimer C, personal communication). This case shows the potential for more serious complications with neurostimulators.

1 Mannheimer C, Carlsson C-A, Ericson K, Vedin A Wiihelmson C. Transcutaneous electrical nerve stimulation in severe angina pectoris. Eur Heart $\mathcal{f} 1982 ; 3$ 297-302.

2 West $P$, Colquhoun $D$. TENS in refractory angina pectoris Med $\mathcal{F}$ A ust 1993;158:488-9.

3 Sanderson J. Electrical neurostimulators for pain relief in angina. Br Heart F 1990;63:141-3. 